\title{
Chemokine Actions in the CNS: Insights from Transgenic Mice
}

\author{
Marcus Müller and Iain L. Campbell
}

\section{Introduction}

Historically the central nervous system (CNS) has been viewed as a relatively immune sheltered tissue. Under physiological conditions the CNS is devoid of leukocytes, including professional antigen presenting cells (APC), is deficient in key immune accessory molecules such as major histocompatibility molecules (MHC) and is protected by an effective blood brain barrier. Significantly, however, in numerous pathological states including infectious diseases and autoimmune disorders (e.g. multiple sclerosis) immune cells are effectively recruited to and infiltrate in the CNS. This immune response can be a two-edged sword required on the one hand to control infection and facilitate tissue repair and regeneration but on the other causing tissue injury that can result in life threatening complications. Therefore, understanding the mechanisms that control the trafficking of leukocytes to the CNS and the subsequent interactions between these cells that contribute to tissue injury has significant implications.

Key to our understanding of the mechanisms that govern leukocyte trafficking has been the relatively recent discovery of a large super-family of mostly small proteins (termed chemokines) that are leukocyte chemoattractant molecules (for reviews see (Rot and von Andrian, 2004; Charo and Ransohoff, 2006). The chemokines are grouped in four subfamilies, $\mathrm{CXC}, \mathrm{CC}, \mathrm{C}$, and $\mathrm{CX} 3 \mathrm{C}$, based on the arrangement of $\mathrm{NH} 2$-terminal cysteine amino acids. It is now clear that chemokines have major roles in regulating leukocyte migration to the brain in inflammatory disease states (Engelhardt, 2006; Rebenko-Moll et al., 2006). The chemokine gene super-family can be further divided into two broad groups based on their gene expression properties. The first group are the constitutively expressed chemokines of which the CXC family chemokine CXCL12 (SDF-1) and the CX3C family member CX3CL1 (fractalkine) are the best characterized. In contrast to the constitutively expressed chemokines, a second larger group of chemokines consisting of many members of both the CXC and CC families, are not detectable under normal conditions in most non-immune tissues including the brain. However, abundant CNS production of chemokines belonging to this second group is found following a variety of insults including trauma, ischemia, infection and inflammation. The induction of these 
chemokines following such insults can be mediated by a number of factors including microbial products (e.g., LPS) and host defense molecules such as cytokines (e.g., IL-1, IFN- $\gamma$ and TNF). Cells intrinsic to the nervous system, including neurons, the macroglia and microglia, all have the ability to produce different chemokines (reviewed in Asensio and Campbell (1999) and Ubogu et al. (2006)). Moreover, the surfaces of these same cells are decorated with a variety of different chemokine receptors. Thus, the CNS seemingly has its own chemokine ligand/receptor network, which is consistent with the growing awareness that the function of chemokines could extend well beyond the regulation of leukocyte trafficking in the brain (Asensio and Campbell, 1999; Mennicken et al., 1999).

So it can be seen that the CNS biology of the chemokines is likely to extend well beyond the singular function of leukocyte chemotaxis. A number of important questions are therefore raised concerning the functions of the chemokines in the CNS. Some key questions include: (1) Do the presence of chemokines in different disordered states reflect their primary involvement or is an epiphenomenon? (2) Is there a cause and effect relationship between the production of a particular chemokine (or chemokines) and the development of specific molecular and cellular neuropathological alterations? (3) What are the mechanisms that underlie chemokinemediated actions in the CNS? (4) Could manipulation of the chemokines or their receptors be used as a rational means for therapeutic intervention? To begin to address these questions experimental approaches are required that are non-invasive and organism-based. Of particular significance in this regard has been the application of genetic engineering technology that has permitted the germline transmission of foreign genes (transgenes) in the mouse. This is accomplished via a fusion gene construct (transgene) in which the gene of interest is placed under the transcriptional control of a cell-specific promoter. The transgene is then introduced (most commonly by microinjection) into the pronucleus of fertilized eggs, which are then implanted in the oviduct of pseudopregnant recipient mice. The transgenic approach has a number of advantages over other approaches such as intracerebral infusion or in vitro cell culture. Transgenic modeling allows the prolonged, reproducible delivery of a specific pure protein to specific cells in the intact CNS. The actions of the transgene product are exerted and can be assessed in a milieu where the anatomic and physiologic interactions of the CNS are preserved. Moreover, individual lines of mice in which the transgene is stably integrated into the genome can be developed and provide an unrestricted source of animals identical for the introduced genetic and molecular alteration, hence permitting systematic multi-level analysis of pathological, electrophysiological, neuroendocrinological and behavioral manifestations. For more detailed reviews of these techniques see (Galli-Taliadoros et al., 1995; Campbell and Gold, 1996; Campbell et al., 1998).

The application of genetically-manipulated animal models to study the CNS biology of the chemokines and their receptors has emerged as an important experimental approach. In this chapter, we will discuss findings from studies based on the use of CNS-chemokine transgenic models. In particular, we will focus on the contribution that these models have made to our understanding of the basic functions and mechanisms of actions of chemokines in the CNS. 


\section{Transgenic Production of Chemokines in the CNS}

As mentioned in the preceding, a great many chemokines belonging in particular to the CXC and CC families are known to be induced in the brain during a variety of different pathologic states (Asensio and Campbell, 1999; Ransohoff, 2002; Rebenko-Moll et al., 2006; Ubogu et al., 2006). The consequences of chemokine expression in the unmanipulated CNS and in some cases in stimulus-evoked disease models have been examined in transgenic mice with CNS-targeted expression of specific chemokines. A summary of the salient features of transgenic models in which CNS-specific promoter constructs were used to drive expression of a specific chemokine gene is given in Table 1 and will be outlined in more detail in the following commentary.

\section{$2.1 C X C L 1(G R O-\alpha / K C / N 51)$}

The chemokine CXCL1 (also known as GRO- $\alpha / \mathrm{KC} / \mathrm{N} 51$ ) contains an N-terminal ELR-amino acid motif and belongs to the CXC-chemokine family. CXCL1 binds to the CXCR2 receptor found predominantly on the surface of neutrophils. Not surprisingly, CXCL1 is a potent neutrophil chemoattractant. That this chemokine has a wider function in the CNS is supported by numerous studies. Cells intrinsic to the CNS including neurons (Horuk et al., 1997), astrocytes (Danik et al., 2003; Flynn et al., 2003), microglia (Filipovic et al., 2003; Flynn et al., 2003), oligodendrocyte precursor cells (Nguyen and Stangel, 2001) and mature oligodendrocytes (Omari et al., 2006) have been reported to have the CXCR2 receptor. A role for CXCR2/ CXCL1 interaction is documented in rodents in the development and maintenance of the oligodendrocyte lineage, myelination, and white matter in the neonatal and adult CNS (Padovani-Claudio et al., 2006).

The CNS function of this chemokine was examined in transgenic mice by placing the CXCL1 gene under the transcriptional control of the myelin basic protein (MBP) promoter (Tani et al., 1996). In the resulting transgenic mice (termed MBP$\mathrm{KC}$ ) transgene-encoded mRNA levels were coincident with the developmentallyregulated pattern of endogenous MBP, peaking at 2-3 week of age, before declining to low levels characteristically seen in adult brain. During the period of maximal transgene expression, the brain of MBP-KC mice was heavily infiltrated with neutrophils that accumulated in perivascular, meningeal and parenchymal sites. At this time, despite loss of blood brain barrier (BBB) integrity and focal gliosis, there was no evidence of brain tissue destruction nor did affected animals display abnormal neurological signs. These results underscore the ability of CXCL1 to promote neutrophil migration to and extravasation in the CNS. However, the absence of any tissue destruction suggests that this chemokine does not activate the neutrophils.

Curiously, in one high transgene expressing line, from $>40$ days of age, mice exhibited a syndrome of progressive neurological dysfunction consisting of slowing 


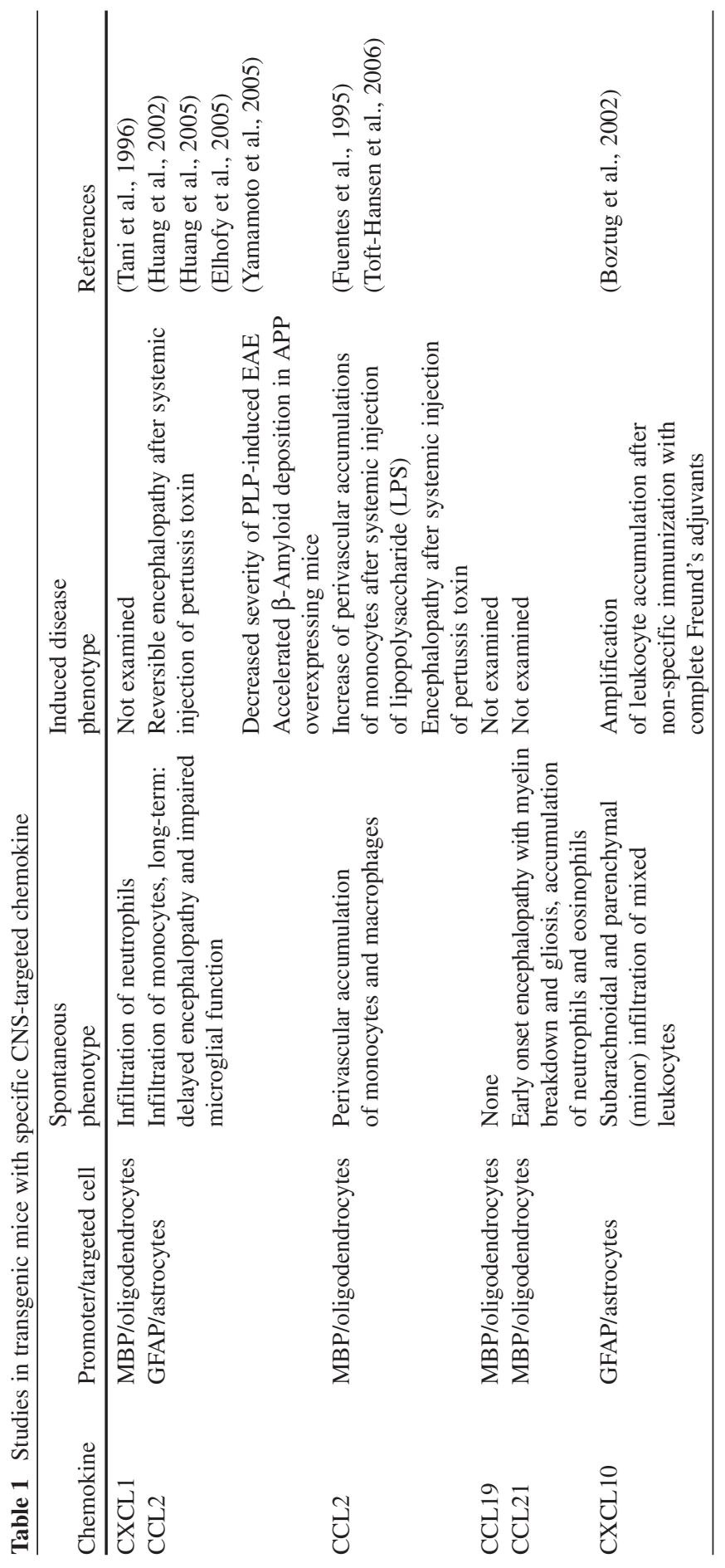


of the righting reflex, clumsiness, increase of rigidity of the hindlimbs and tail and profound truncal instability. The major neuropathological findings were florid microglial activation and BBB disruption without dysmyelination. Despite the severity of this neurological disorder (which resulted in premature death), there was no histological evidence of damage to neurons, myelin or axons. Although the basis for the neurological dysfunction developing in the MBP-KC mice is unknown, Tani et al. (1996) speculated that this might be due to the glial and BBB perturbations. It is also possible as the authors suggested that chronic exposure of neurons to the chemokine may directly compromise their function. In support of such a mechanism, CXCL1 can enhance both stimulus-evoked and spontaneous postsynaptic currents (Ragozzino et al., 1998), and increase neurotransmitter release and reduce long-term depression (Giovannelli et al., 1998), in Purkinje neurons. On the other hand, despite accumulating evidence that CXCL1 influences oligodendrocyte precursor cell and differentiated oligodendrocyte function both in vitro and in vivo, surprisingly, MBP-KC mice were reported to exhibit normal CNS myelination both in the acute and chronic disorders (Tani et al., 1996).

\subsection{CXCL10 (IP-10)}

CXCL10 (also known as IP-10) belongs to a sub-family of chemokines that include CXCL9 (MIG) and CXCL11 (ITAC) within the CXC family. Unlike CXCL1, the chemokines in this sub-family lack the N-terminal ELR-amino acid motif. Expression of the CXCL9, CXCL10 and CXCL11 genes is induced by the cytokine IFN- $\gamma$ and all three chemokines bind to a common receptor, CXCR3. High levels of CXCR3 can be found principally on activated CD4 ${ }^{+}$Th1 and CD8 ${ }^{+}$T-cells and NK-cells (Taub et al., 1993, 1996a; Hopkins and Rothwell, 1995; Liao et al., 1995; Loetscher et al., 1996; Cole et al., 1998). In keeping with this cellular receptor distribution, CXC9, CXCL10 and CXCL11 all promote the trafficking of these immune cells in vitro and are strongly implicated in the generation of type I immune responses associated with anti-viral host defense, transplant rejection and autoimmunity (Liu et al., 2005). Evidence from rodent studies indicate that a functional CXCR3 receptor exists on microglia (Rappert et al., 2004) and neurons (Nelson and Gruol, 2004). Descriptive studies confirm the presence of high levels of CXCL10 RNA and protein, particularly in astrocytes and neurons, in a variety of experimental and human neurological diseases including viral infection and autoimmune diseases (Klein, 2004; Liu et al., 2005).

A transgenic mouse model was developed in which the GFAP promoter was used to accomplish astrocyte-targeted production of CXCL10 (see Fig. 10.1) (Boztug et al., 2002). Astrocyte-localized production of the GFAP-transgene encoded CXCL10 RNA was confirmed while production of CXCL10 at the protein level was demonstrated by immunoblotting. The levels of these transgene-encoded products were shown to be similar to levels of the endogenous CXCL10 induced following viral infection of the mouse brain confirming the 


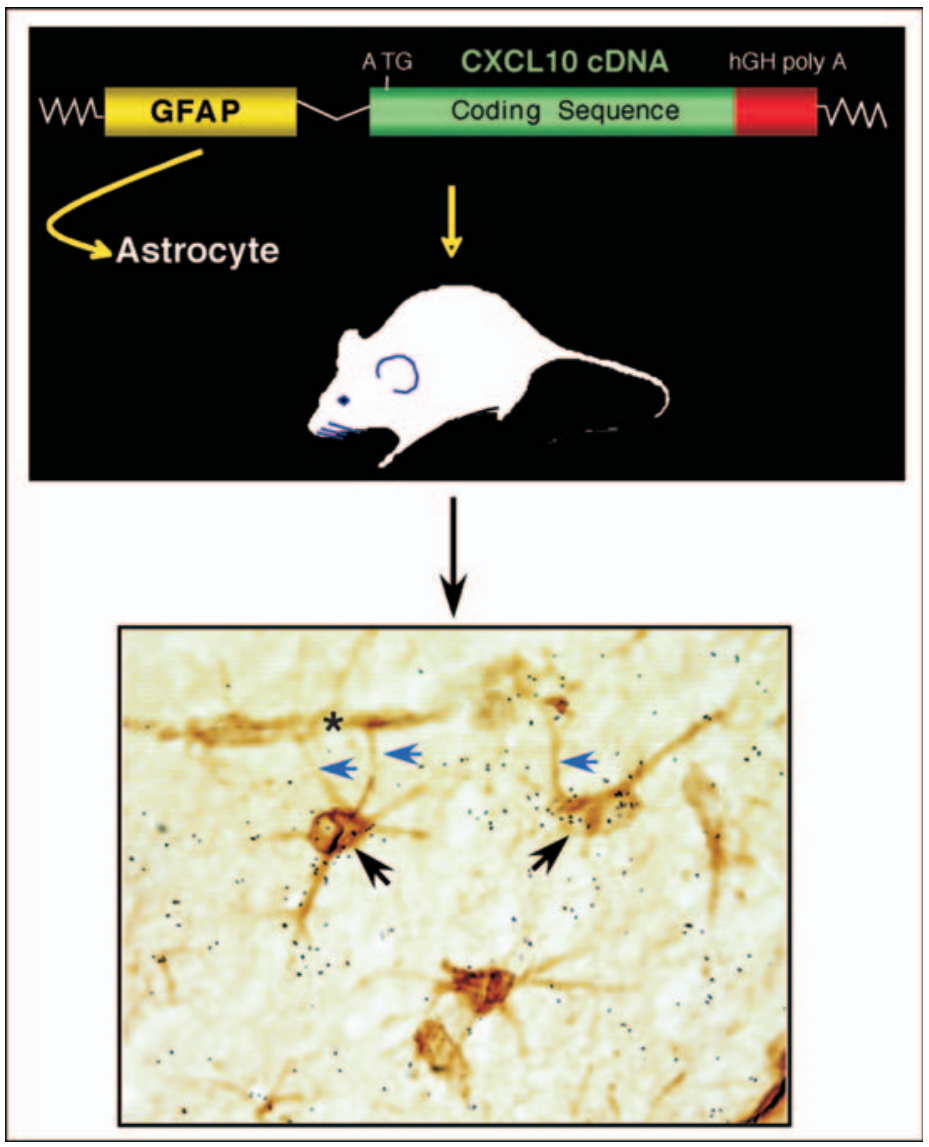

Fig. 10.1 Generation of transgenic mice with CXCL10 gene production targeted to astrocytes. A cDNA encoding murine CXCL10 was inserted downstream of the murine GFAP promoter and upstream of a human growth hormone (hGH) polyadenylation signal sequence. This transgene construct was microinjected into the germ line of mice to generate a stable transgenic line. The presence of CXCL10 gene expression in astrocytes (lower panel) was confirmed by in situ hybridization for CXCL10 RNA combined with immunohistochemistry for GFAP protein. Note that some CXCL10 RNA positive astrocytes (black arrows) extend processes (blue arrows) to a blood vessel (asterisk) that is close by (See Color Plates).

pathophysiological relevance of this model. These GFAP-CXCL10 transgenic mice bred normally and appeared physically unimpaired. Histological evaluation of the brain revealed a predominantly subarachnoidal and meningeal accumulation of mixed leukocytes with only minor parenchymal infiltration (see Fig. 10.2). The accumulation of leukocytes in the brain of these transgenic mice was markedly amplified by systemic immune challenge after immunization with complete Freund's adjuvant and pertussis toxin. Immunophenotypic characterization of these infiltrates revealed surprisingly, that the majority of infiltrating leukocytes 


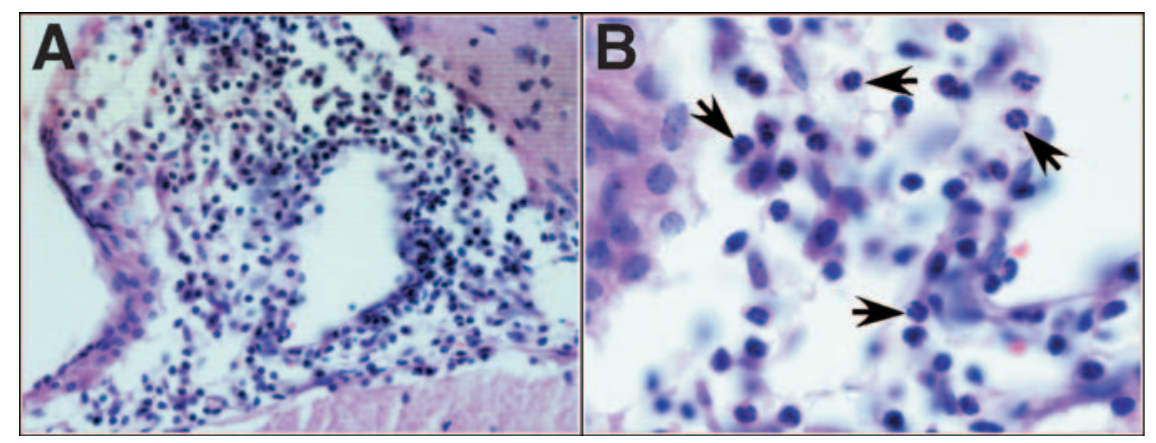

Fig. 10.2 Histological evaluation of the brain from GFAP-CXCL10 transgenic mice. A. appearance of a perivascular leukocyte infiltrate in the meninges (haematoxylin and eosin stained section; original magnification $\times 100$ ). B. numerous neutrophils were observed (arrows) in the leukocyte infiltrates, (haematoxylin \& eosin stained section; original magnification $\times 1,000)($ See Color Plates).

were neutrophils and macrophages with a minority being represented by $\mathrm{CD}^{+} \mathrm{T}-$ cells. These observations suggest that constitutive astrocyte production of CXCL10 in the CNS does not provide an effective signal for the recruitment of T-cells from the periphery. This conclusion is in keeping with the findings from more recent reports that found that mice deficient for CXCL10 exhibit similar clinical and pathological outcomes as wild type controls for experimental autoimmune encephalomyelitis (EAE-a CD4 + T-cell-mediated disease) (Klein et al., 2004) as well as murine hepatitis virus encephalitis (Stiles et al., 2006).

The finding that neutrophils were preferentially recruited to the CNS of the GFAP-CXCL10 transgenic mice was unexpected since these cells were not known to have CXCR3. This point was confirmed by the studies of Boztug et al. (2002) who used fluorescence activated cell sorting analysis to show that the neutrophil population present in the CNS of the GFAP-CXCL10 transgenic mice were CXCR3 negative. Moreover, the results of a survey of the cerebral expression of a large number of other chemokines including ELR-CXC chemokines known to have neutrophil chemoattractant properties proved unremarkable. Therefore, the basis for the preferential accumulation of neutrophils in the brain of the transgenic mice is unknown. In all, these findings argue for a CXCR3-independent chemoattractant mechanism mediated by CXCL10 for the recruitment of neutrophils to the brain. It is interesting to note that an alternative receptor for CXCL10 has been reported on endothelial and epithelial cells (Soejima and Rollins, 2001). However, the identity of this novel CXCL10 binding moiety and its functional signature is currently unknown.

Of further note in these GFAP-CXCL10 transgenic mice was the lack of any gliosis or degenerative features that would be expected if there existed a destructive immunoinflammatory response in the CNS. So while constitutive, astrocyte-targeted production of CXCL10 can promote the recruitment of leukocytes to the CNS, this chemokine lacks the ability to further influence these cells, in particular, to drive 
a functional immune response. Moreover, although there are reports that a functional CXCR3 exists on neurons (Nelson and Gruol, 2004) and microglia (Rappert et al., 2004) in the rodent CNS, there would seem to be little effect of CXCL10 on these cells in this transgenic model. A possible explanation for this lack of effect of CXCL10 is that there is desensitization to the chemokine through CXCR3 downregulation. In support of this idea, hippocampal slices from wild type but not GFAP-CXCL10 transgenic mice in response to acute exposure to recombinant CXCL10 protein were shown to exhibit altered synaptic plasticity that was CXCR3dependent (Vlkolinsky et al., 2004).

Interestingly, the role of CXCL10 as an effective T-cell chemoattractant may depend on the tissue in which the chemokine is produced. In contrast to the CNS, transgenic production of CXCL10 targeted to the beta cells of the islets of Langerhans induced spontaneous infiltration of large numbers of CD4 + and CD8 + T-cells around the islets (Rhode et al., 2005). Moreover, islet CXCL10 production markedly accelerated a stimulus-evoked autoimmune process by enhancing the migration of antigen-specific T-cells to the islets resulting in the development type I diabetes.

\subsection{CCL2 (MCP-1)}

The chemokine CCL2 (also known as MCP-1 and JE) was first identified as a monocyte attracting chemokine (Rollins, 1991; Rollins et al., 1991) but also has been shown to attract a wide variety of other leukocytes, including, activated T-cells, dendritic cells, mast cells and basophils (Taub et al., 1996b; Gunn et al., 1997; Siveke and Hamann, 1998). Although CCL2 binds to a number of CC receptors the primary receptor for this chemokine is CCR2. Cells within the CNS including astrocytes and microglia have been shown to be positive for CCR2 (Banisadr et al., 2002), while the ligand is produced by astrocytes (Ransohoff et al., 1993; Glabinski et al., 1996). CCL2 is implicated in the pathogenesis of a wide variety of experimental and human neurodegenerative and immune-mediated disorders (Cartier et al., 2005).

The first transgenic model developed to achieve CCL2 production in the CNS of mice employed the MBP-promoter to drive expression of the CCL2 gene in oligodendrocytes (Fuentes et al., 1995). Temporal expression of transgene-encoded CCL2 RNA followed that of endogenous MBP peaking between the second and third post-natal week before declining. In addition, CCL2 protein was demonstrated to be present in the brain of the transgenic but not wild type mice during peak transgene gene expression. In concordance with the temporal profile of transgene-encoded CCL2 production, discrete monocytic cell infiltrates were observed throughout the brain predominantly in perivascular sites in the meninges, choroid plexus, and brain parenchymal white matter. The intensity of these infiltrates could be increased as well as further parenchymal infiltration stimulated, following systemic 
LPS administration to the MBP-MCP1 transgenic mice. Importantly, there was no evidence of either spontaneous or stimulus-evoked CNS injury, particularly in white matter, in these transgenic mice. The observations in this model confirmed the potent monocyte chemoattractant properties of CCL2 and further indicate that while CCL2 can provide a signal for the recruitment of these cells to the CNS, the chemokine does not directly stimulate these cells to engage in an active inflammatory process.

However, a more recent study using these transgenic mice revealed that a systemic injection of pertussis toxin can induce a reversible encephalopathy with clinical signs, including tremor, inactivity, limb clasping and weight loss (ToftHansen et al., 2006). Encephalopathic signs were correlated with a more diffuse distribution of monocytes, which were less restricted to the perivascular space suggesting a role for the inflammatory process in the clinical symptoms. However the pathological basis for the physical impairments in these mice was not determined and it therefore remains unclear whether tissue injury was also involved. Increased levels of metalloproteinases were found in pertussis toxin treated MBP-CCL2 transgenic mice, suggesting a critical need for metalloproteinases for the parenchymal infiltration of the monocytes. Weight loss and parenchymal infiltration, but not perivascular accumulation, were markedly resolved by the broad-spectrum metalloproteinase inhibitor BB-94/Batimastat. The findings suggest that a critical event in inducing disease in this model is metalloproteinase-dependent leukocyte migration across the astroglial basement membrane of the blood-brain barrier, which is induced by pertussis toxin.

As noted above, astrocytes but not oligodendrocytes have been found to be a major source of CCL2 in various CNS pathologies. The production of CCL2 under the control of the MBP-promoter follows a similar temporal pattern as the endogenous gene and produces a spike of CCL2 activity. The delivery of maximal transgene-derived CCL2 in this model occurs during a developmentally sensitive period before subsiding making this model less relevant for studying the effects of CCL2 on the adult brain. In order to address these issues, Huang and co-workers generated transgenic mice with astrocyte-targeted expression of the CCL2 gene driven by the human GFAP promoter (Huang et al., 2002, 2005). These GFAPCCL2 transgenic mice produced CCL2 in the CNS at levels similar to those produced during EAE, yet, these animals exhibited little spontaneous inflammation. This contrasts with the MBP-CCL2 transgenic mouse model in which widespread and marked perivascular accumulation of monocytes was seen. The explanation for this difference between these models is not clear but it may reflect the chronic and more generalized production of high levels of CCL2 in the case of the GFAPCCL2 transgenic mice. However, systemic immunization with complete Freund's adjuvant and injection of pertussis toxin induces a transient encephalopathy with elevated levels of IFN- $\gamma$ and IL- 2 in the GFAP-CCL2 transgenic mice. Interestingly, the encephalopathy was less severe in CCL2 transgenic mice on a T-cell deficient background, indicating the involvement of T-cells and not only monocytes in the observed encephalopathy (Huang et al., 2002). Moreover, this phenotype was 
shown to be dependent on CCR2 indicating that under these conditions CCR2 is the principal receptor for CCL2 in the murine CNS. Although a number of studies have suggested that CCR2 activation in the periphery downregulates Th1-T-cell development and can augment Th2-T-cell development (Daly and Rollins, 2003), the findings in the GFAP-CCL2 transgenic mouse indicate that in the CNS, CCL2 may recruit Th1 T-cells or direct T-cell polarization toward type 1 cytokine production. This concept is also supported by the finding that mice deficient for the CCL2 receptor, CCR2, are resistant to the development of the CD4 ${ }^{+} \mathrm{T}$-cell-mediated autoimmune disease, EAE (Fife et al., 2000). However, in findings that are difficult to reconcile with these conclusions, it has been reported that GFAP-CCL2 transgenic mice developed significantly milder EAE disease than littermate controls (Elhofy et al., 2005). Antigen-specific T-cells recovered from the GFAP-CCL2 transgenic mice showed decrease proliferative response to autoantigen and secreted less IFN- $\gamma$ and had lower levels of IL-12 receptor RNA. These studies suggest that rather than augmenting Th1 cells, that chronic CCL2 production in the CNS impairs the function of these cells.

Although it has been possible to produce stimulus-evoked encephalopathy in MBP- and GFAP-CCL2 transgenic mice by systemic innate immune challenge these disorders are largely transient and therefore do not model the increased levels of CNS CCL2 that have been reported to occur in chronic neurological diseases such as human immunodeficiency virus type 1-associated dementia, amyotrophic lateral sclerosis, and multiple sclerosis. However, Huang et al. (2005) report from a more recent study that GFAP-CCL2 transgenic mice develop a spontaneous, delayed encephalopathy from $>7$ months of age. These mice presented with loss of weight, a hunched posture, slow response to tactile stimuli and a flaccid tail. Some animals even developed a hind-limb paralysis at $>12$ months of age. The brain exhibited perivascular infiltrates made up of activated macrophages, microglia with morphological activation, and impaired BBB. Surprisingly, no evidence was found for demyelination or of reduced neurons, axons, or synapses of neural components. Microglia in the GFAP-CCL2 transgenic mice, despite the appearance of activation and increased CD45 immunoreactivity, were found to be defective in their response to environmental stimuli in vitro. The Authors propose that chronic elevation of CCL2 in the CNS leads to desensitization of CCR2 and microglial dysfunction.

Further support for the notion that chronic CCL2 leads to microglial dysfunction comes from a study by Yamamoto et al. who generated bigenic GFAP-CCL2/tg 2576 mice that, in addition to chronic CCL2, also overproduce a mutant amyloid precursor protein (APP) in the CNS (Yamamoto et al., 2005). These workers found a significant increase in amyloid deposition in CCL2/APP bigenic mice compared with APP singly transgenic mice. Despite being accompanied by an increase in lesion-associated macrophages/microglia, evidence is provided suggesting that these cells show reduced phagocytic function leading to accelerated amyloid deposition. These findings underline the importance of considering the participation of chemokines and chemokine driven monocyte accumulation and function in the pathogenesis of degenerative CNS diseases. 


\subsection{CCL19 (MIP-3ß/ELC/exodus-3) and CCL21 (6Ckine/.SLC/exodus-2)}

The chemokines CCL19 and CCL21 have been implicated in the migration of lymphocytes and are produced in secondary lymphoid organs such as the lymph nodes. Both these chemokines bind to CCR7 and CCR11. Mice lacking CCR7 have impaired migration of lymphocytes into secondary lymphoid organs (Forster et al., 1999). CCL21 but not CCL19 has also been reported to bind to CXCR3 (Rappert et al., 2002). CCL19 and CCL21 are localized in venules surrounded by CCR7 positive inflammatory cells in the brain and spinal cord of mice with EAE and functional studies suggest that CCL19 and CCL21 are involved in $\mathrm{T}$ lymphocyte migration into the central nervous system during EAE (Alt et al., 2002). However, recent studies in CCR7 deficient mice suggest that CCL19 and CCL21 function may be dispensable in EAE (Pahuja et al., 2006). This interpretation is complicated in the case of CCL2 1 since this chemokine could still play a role in EAE in CCR7 deficient mice via CXCR3 on encephalitogenic T-cells. Moreover, CCL21 was reported to be produced by neurons in the murine brain following ischemic insult, highlighting the existence of an intrinsic source of this chemokine in the CNS that could interact with microglia via CXCR3 (Biber et al., 2002).

To study the biological role of the chemokine ligands CCL19 and CCL21, transgenic mice were developed that expressed either CCL19 or CCL21 in oligodendrocytes of the CNS using an MBP-promoter construct (Chen et al., 2002a). As expected, transgene-encoded CCL19 and CCL20 mRNA was developmentally regulated and peaked at 2-3 weeks of age. Expression of CCL19 or CCL21 protein in the CNS of the transgenic mice was confirmed by immunohistochemical staining. CCL19 producing transgenic mice did not show any clinical or histological phenotype, which led to the conclusion that CCL19, at least from diffuse cerebral expression, is not able to attract leukocytes into the brain or cause a CNS pathology. In contrast, transgenic mice with MBP-promoter driven production of CCL21 developed a rapid onset encephalopathy with tremor, ataxia and weight loss. Most of the CCL21 producing animals died within 4 weeks. Studies in vitro documented a chemotactic effect of CCL21 for thymocytes, naïve T cells, dendritic cells and B cells and not for macrophages and neutrophils (Hedrick and Zlotnik, 1997; Nagira et al., 1997; Kellermann et al., 1999). However, cerebral infiltrates in CCL21 producing transgenic mice consisted almost exclusively of neutrophils and eosinophils. These infiltrates were associated with gliosis, microglial activation and demyelination. Infiltrating lymphocytes were not observed-even in areas in which the BBB was disrupted. The lack of lymphocyte infiltration is a notable finding because transgenic expression of CCL21 in the pancreas induced lymphocyte influx and led to the formation of lymph node like structures (Fan et al., 2000; Chen et al., 2002b). It would thus appear that tissue-specific factors that might include for example, specific adhesion molecules and other cofactors, are required for CCL21 to induce lymphocyte recruitment. 
As pointed out by the Authors the phenotypic differences observed between MBP-CCL21 and MBP-CCL19 transgenic mice is very interesting given that these chemokines are functionally related and bind to the same cell surface receptors, CCR7 and CCR11. However, and as noted above CCL21 but not CCL19 also binds to CXCR3, which in the case of murine microglia produces functional alterations in these cells (Rappert et al., 2002). Interestingly, the same workers have reported that neurons in the ischemic brain produce CCL21 (Biber et al., 2001). These observations have led to the suggestion that CCL21 and CXCR3 may mediate neuron-glia interactions during disease conditions. It is speculated that CCL21 derived from transgenic oligodendrocytes in the brain of the MBP-CCL21 mice may have interacted with CXCR3 on microglia inducing an inflammatory response that led to the influx of inflammatory cells into the CNS. While further studies, such as crossing the MBP-CCL21 transgenic mice with CXCR3 deficient animals, would help to clarify this mechanism, as noted in the preceding discussion, transgenic mice with astrocyte-targeted production of the primary CXCR3 ligand CXCL10 do not share the severe neurological phenotype of the MBP-CCL2 mice.

\section{Concluding Discussion}

An important constraint with the transgenic approach that needs to be considered relates to the spatial and temporal control of transgene expression. The production of chemokines in pathologic states such as in EAE is regulated in a temporal fashion that precedes and then overlaps with the severity of the disease process. Moreover, the spatial pattern of expression of chemokines such as CXCL10 is focally restricted to the immunoinflammatory lesions. The production of chemokines achieved in pathologic states therefore would favor the establishment of highly localized chemoattractant gradients that then guide the trafficking of leukocytes. In contrast, in the transgenic model the chemokine is produced more chronically and more diffusely throughout the CNS. In addition to the potential for receptor down-regulation and desensitization of the ligand response, it is likely that any chemoattractant gradient in this milieu would be much weaker in strength and more widely distributed. Technically achieving more acute and focal expression of the chemokine to better replicate pathologic states in the adult brain using transgenic modeling is clearly desirable but difficult to achieve at this time. Although constructs have been engineered that permit the temporal control of transgene expression in the CNS, more precise spatial targeting that would allow for highly localized production of the chemokine in neural cells such as astrocytes is not yet possible.

Despite the drawbacks noted in the preceding discussion, it is clear that development of molecular genetic methods that allow for the targeted manipulation of gene expression in an intact organism has been important in contributing to our understanding of the actions of chemokines in the CNS in physiologic and pathologic states (see Table 1). Promoter-driven expression of different biologically relevant chemokines in the CNS of transgenic mice highlight the ability of some but not all 
of these molecules to stimulate the recruitment but commonly not the functional activation of specific subsets of leukocytes to the CNS. With certain chemokines such as CXCL10 and CCL21, their transgenic production in the CNS led to the accumulation of unexpected leukocyte phenotypes that could not have been predicted based on either in vitro chemotaxis assays or the known cellular distribution of the receptor. This to some extent reflects the complexity of the actions and interactions between chemokines and their receptors many of which we know to be promiscuous for the ligands they bind. Moreover, differences in the immunophenotype of leukocytes recruited to the CNS versus peripheral tissues following organspecific transgene driven chemokine production indicates that tissue-specific influences can clearly determine how a chemokine will behave in that milieu. There are still considerable gaps in our understanding of chemokine biology and neurobiology. However, ongoing and future studies employing approaches such as transgenic modeling no doubt will begin to fill these gaps.

Acknowledgements The authors studies referred to in this article were funded by USPHS grants MH 62231 and NS044905.

\section{References}

Alt, C., Laschinger, M. and Engelhardt, B. (2002). Functional expression of the lymphoid chemokines CCL19 (ELC) and CCL 21 (SLC) at the blood-brain barrier suggests their involvement in G-protein-dependent lymphocyte recruitment into the central nervous system during experimental autoimmune encephalomyelitis. Eur J Immunol, 32, 2133-2144.

Asensio, V. C. and Campbell, I. L. (1999). Chemokines in the CNS: plurifunctional mediators in diverse states. Trends Neurosci, 22, 504-512.

Banisadr, G., Queraud-Lesaux, F., Boutterin, M. C., Pelaprat, D., Zalc, B., Rostene, W., Haour, F. and Parsadaniantz, S. M. (2002). Distribution, cellular localization and functional role of CCR2 chemokine receptors in adult rat brain. J Neurochem, 81, 257-269.

Biber, K., Sauter, A., Brouwer, N., Copray, S. C. and Boddeke, H. W. (2001). Ischemia-induced neuronal expression of the microglia attracting chemokine secondary lymphoid-tissue chemokine (SLC). Glia, 34, 121-133.

Biber, K., Dijkstra, I., Trebst, C., De Groot, C. J., Ransohoff, R. M. and Boddeke, H. W. (2002). Functional expression of CXCR3 in cultured mouse and human astrocytes and microglia. Neuroscience, 112, 487-497.

Boztug, K., Carson, M. J., Pham-Mitchell, N., Asensio, V. C., DeMartino, J. and Campbell, I. L. (2002). Leukocyte infiltration, but not neurodegeneration, in the CNS of transgenic mice with astrocyte production of the CXC chemokine ligand 10. J Immunol, 169, 1505-1515.

Campbell, I. L. and Gold, L. H. (1996). Transgenic modeling of neuropsychiatric disorders. Mol Psychiatry, 1, 105-120.

Campbell, I. L., Stalder, A. K., Akwa, Y., Pagenstecher, A. and Asensio, V. C. (1998). Transgenic models to study the actions of cytokines in the central nervous system. Neuroimmunomodulation, $5,126-135$.

Cartier, L., Hartley, O., Dubois-Dauphin, M. and Krause, K. H. (2005). Chemokine receptors in the central nervous system: role in brain inflammation and neurodegenerative diseases. Brain Res Brain Res Rev, 48, 16-42.

Charo, I. F. and Ransohoff, R. M. (2006). The many roles of chemokines and chemokine receptors in inflammation. $N$ Engl J Med, 354, 610-621.

Chen, S. C., Leach, M. W., Chen, Y., Cai, X. Y., Sullivan, L., Wiekowski, M., Dovey-Hartman, B. J., Zlotnik, A. and Lira, S. A. (2002a). Central nervous system inflammation and neurological 
disease in transgenic mice expressing the CC chemokine CCL21 in oligodendrocytes. J Immunol, $168,1009-1017$.

Chen, S. C., Vassileva, G., Kinsley, D., Holzmann, S., Manfra, D., Wiekowski, M. T., Romani, N. and Lira, S. A. (2002b). Ectopic expression of the murine chemokines CCL21a and CCL21b induces the formation of lymph node-like structures in pancreas, but not skin, of transgenic mice. J Immunol, 168, 1001-1008.

Cole, K. E., Strick, C. A., Paradis, T. J., Ogborne, K. T., Loetscher, M., Gladue, R. P., Lin, W., Boyd, J. G., Moser, B., Wood, D. E., Sahagan, B. G. and Neote, K. (1998). Interferon-inducible $\mathrm{T}$ cell alpha chemoattractant (I-TAC): a novel non-ELR CXC chemokine with potent activity on activated $\mathrm{T}$ cells through selective high affinity binding to CXCR3. J Exp Med, 187, 2009-2021.

Daly, C. and Rollins, B. J. (2003). Monocyte chemoattractant protein-1 (CCL2) in inflammatory disease and adaptive immunity: therapeutic opportunities and controversies. Microcirculation, 10, 247-257.

Danik, M., Puma, C., Quirion, R. and Williams, S. (2003). Widely expressed transcripts for chemokine receptor CXCR1 in identified glutamatergic, gamma-aminobutyric acidergic, and cholinergic neurons and astrocytes of the rat brain: a single-cell reverse transcription-multiplex polymerase chain reaction study. J Neurosci Res, 74, 286-295.

Elhofy, A., Wang, J., Tani, M., Fife, B. T., Kennedy, K. J., Bennett, J., Huang, D., Ransohoff, R. M. and Karpus, W. J. (2005). Transgenic expression of CCL2 in the central nervous system prevents experimental autoimmune encephalomyelitis. J Leukoc Biol, 77, 229-237.

Engelhardt, B. (2006). Molecular mechanisms involved in T cell migration across the blood-brain barrier. J Neural Transm, 113, 477-485.

Fan, L., Reilly, C. R., Luo, Y., Dorf, M. E. and Lo, D. (2000). Cutting edge: ectopic expression of the chemokine TCA4/SLC is sufficient to trigger lymphoid neogenesis. J Immunol, 164, 3955-3959.

Fife, B. T., Huffnagle, G. B., Kuziel, W. A. and Karpus, W. J. (2000). CC chemokine receptor 2 is critical for induction of experimental autoimmune encephalomyelitis. $J$ Exp Med, 192, 899-905.

Filipovic, R., Jakovcevski, I. and Zecevic, N. (2003). GRO-alpha and CXCR2 in the human fetal brain and multiple sclerosis lesions. Dev Neurosci, 25, 279-290.

Flynn, G., Maru, S., Loughlin, J., Romero, I. A. and Male, D. (2003). Regulation of chemokine receptor expression in human microglia and astrocytes. J Neuroimmunol, 136, 84-93.

Forster, R., Schubel, A., Breitfeld, D., Kremmer, E., Renner-Muller, I., Wolf, E. and Lipp, M. (1999). CCR7 coordinates the primary immune response by establishing functional microenvironments in secondary lymphoid organs. Cell, 99, 23-33.

Fuentes, M. E., Durham, S. K., Swerdel, M. R., Lewin, A. C., Barton, D. S., Megill, J. R., Bravo, R. and Lira, S. A. (1995). Controlled recruitment of monocytes and macrophages to specific organs through transgenic expression of monocyte chemoattractant protein-1. J Immunol, 155, $5769-5776$.

Galli-Taliadoros, L. A., Sedgwick, J. D., Wood, S. A. and Korner, H. (1995). Gene knock-out technology: a methodological overview for the interested novice. J Immunol Methods, 181, $1-15$.

Giovannelli, A., Limatola, C., Ragozzino, D., Mileo, A. M., Ruggieri, A., Ciotti, M. T., Mercanti, D., Santoni, A. and Eusebi, F. (1998). CXC chemokines interleukin-8 (IL-8) and growth-related gene product alpha (GROalpha) modulate Purkinje neuron activity in mouse cerebellum. J Neuroimmunol, 92, 122-132.

Glabinski, A. R., Balasingam, V., Tani, M., Kunkel, S. L., Strieter, R. M., Yong, V. W. and Ransohoff, R. M. (1996). Chemokine monocyte chemoattractant protein-1 is expressed by astrocytes after mechanical injury to the brain. J Immunol, 156, 4363-4368.

Gunn, M. D., Nelken, N. A., Liao, X. and Williams, L. T. (1997). Monocyte chemoattractant protein-1 is sufficient for the chemotaxis of monocytes and lymphocytes in transgenic mice but requires an additional stimulus for inflammatory activation. J Immunol, 158, 376-383. 
Hedrick, J. A. and Zlotnik, A. (1997). Identification and characterization of a novel beta chemokine containing six conserved cysteines. J Immunol, 159, 1589-1593.

Hopkins, S. J. and Rothwell, N. J. (1995). Cytokines and the nervous system. I: expression and recognition [see comments]. Trends Neurosci, 18, 83-88.

Horuk, R., Martin, A. W., Wang, Z., Schweitzer, L., Gerassimides, A., Guo, H., Lu, Z., Hesselgesser, J., Perez, H. D., Kim, J., Parker, J., Hadley, T. J. and Peiper, S. C. (1997). Expression of chemokine receptors by subsets of neurons in the central nervous system. J Immunol, $158,2882-2890$.

Huang, D., Tani, M., Wang, J., Han, Y., He, T. T., Weaver, J., Charo, I. F., Tuohy, V. K., Rollins, B. J. and Ransohoff, R. M. (2002). Pertussis toxin-induced reversible encephalopathy dependent on monocyte chemoattractant protein-1 overexpression in mice. $J$ Neurosci, 22, 10633-10642.

Huang, D., Wujek, J., Kidd, G., He, T. T., Cardona, A., Sasse, M. E., Stein, E. J., Kish, J., Tani, M., Charo, I. F., Proudfoot, A. E., Rollins, B. J., Handel, T. and Ransohoff, R. M. (2005). Chronic expression of monocyte chemoattractant protein-1 in the central nervous system causes delayed encephalopathy and impaired microglial function in mice. FASEB J, 19, 761-772.

Kellermann, S. A., Hudak, S., Oldham, E. R., Liu, Y. J. and McEvoy, L. M. (1999). The CC chemokine receptor-7 ligands 6Ckine and macrophage inflammatory protein-3 beta are potent chemoattractants for in vitro- and in vivo-derived dendritic cells. J Immunol, 162, 3859-3864.

Klein, R. S. (2004). Regulation of neuroinflammation: the role of CXCL10 in lymphocyte infiltration during autoimmune encephalomyelitis. J Cell Biochem, 92, 213-222.

Klein, R. S., Izikson, L., Means, T., Gibson, H. D., Lin, E., Sobel, R. A., Weiner, H. L. and Luster, A. D. (2004). IFN-inducible protein 10/CXC chemokine ligand 10-independent induction of experimental autoimmune encephalomyelitis. J Immunol, 172, 550-559.

Liao, F., Rabin, R. L., Yannelli, J. R., Koniaris, L. G., Vanguri, P. and Farber, J. M. (1995). Human mig chemokine: biochemical and functional characterization. J Exp Med, 182, 1301-1314.

Liu, L., Callahan, M. K., Huang, D. and Ransohoff, R. M. (2005). Chemokine receptor CXCR3: an unexpected enigma. Curr Top Dev Biol, 68, 149-181.

Loetscher, M., Gerber, B., Loetscher, P., Jones, S. A., Piali, L., Clark-Lewis, I., Baggiolini, M. and Moser, B. (1996). Chemokine receptor specific for IP10 and mig: structure, function, and expression in activated T-lymphocytes. J Exp Med, 184, 963-969.

Mennicken, F., Maki, R., de Souza, E. B. and Quirion, R. (1999). Chemokines and chemokine receptors in the CNS: a possible role in neuroinflammation and patterning. Trends Pharmacol Sci, 20, 73-78.

Nagira, M., Imai, T., Hieshima, K., Kusuda, J., Ridanpaa, M., Takagi, S., Nishimura, M., Kakizaki, M., Nomiyama, H. and Yoshie, O. (1997). Molecular cloning of a novel human CC chemokine secondary lymphoid-tissue chemokine that is a potent chemoattractant for lymphocytes and mapped to chromosome 9p13. J Biol Chem, 272, 19518-19524.

Nelson, T. E. and Gruol, D. L. (2004). The chemokine CXCL10 modulates excitatory activity and intracellular calcium signaling in cultured hippocampal neurons. J Neuroimmunol, 156, 74-87.

Nguyen, D. and Stangel, M. (2001). Expression of the chemokine receptors CXCR1 and CXCR2 in rat oligodendroglial cells. Brain Res Dev Brain Res, 128, 77-81.

Omari, K. M., John, G., Lango, R. and Raine, C. S. (2006). Role for CXCR2 and CXCL1 on glia in multiple sclerosis. Glia, 53, 24-31.

Padovani-Claudio, D. A., Liu, L., Ransohoff, R. M. and Miller, R. H. (2006). Alterations in the oligodendrocyte lineage, myelin, and white matter in adult mice lacking the chemokine receptor CXCR2. Glia, 54, 471-483.

Pahuja, A., Maki, R. A., Hevezi, P. A., Chen, A., Verge, G. M., Lechner, S. M., Roth, R. B., Zlotnik, A. and Alleva, D. G. (2006). Experimental autoimmune encephalomyelitis develops in CC chemokine receptor 7-deficient mice with altered T-cell responses. Scand J Immunol, 64, 361-369. 
Ragozzino, D., Giovannelli, A., Mileo, A. M., Limatola, C., Santoni, A. and Eusebi, F. (1998). Modulation of the neurotransmitter release in rat cerebellar neurons by GRO beta. Neuroreport, 9, 3601-3606.

Ransohoff, R. M. (2002). The chemokine system in neuroinflammation: an update. J Infect Dis, 186 Suppl 2, S152-S156.

Ransohoff, R. M., Hamilton, T. A., Tani, M., Stoler, M. H., Shick, H. E., Major, J. A., Estes, M. L., Thomas, D. M. and Tuohy, V. K. (1993). Astrocyte expression of mRNA encoding cytokines IP-10 and JE/MCP-1 in experimental autoimmune encephalomyelitis. FASEB J, 7, 592-600.

Rappert, A., Biber, K., Nolte, C., Lipp, M., Schubel, A., Lu, B., Gerard, N. P., Gerard, C., Boddeke, H. W. and Kettenmann, H. (2002). Secondary lymphoid tissue chemokine (CCL21) activates CXCR3 to trigger a Cl- current and chemotaxis in murine microglia. J Immunol, 168, 3221-3226.

Rappert, A., Bechmann, I., Pivneva, T., Mahlo, J., Biber, K., Nolte, C., Kovac, A. D., Gerard, C., Boddeke, H. W., Nitsch, R. and Kettenmann, H. (2004). CXCR3-dependent microglial recruitment is essential for dendrite loss after brain lesion. J Neurosci, 24, 8500-8509.

Rebenko-Moll, N. M., Liu, L., Cardona, A. and Ransohoff, R. M. (2006). Chemokines, mononuclear cells and the nervous system: heaven (or hell) is in the details. Curr Opin Immunol, 18, 683-689.

Rhode, A., Pauza, M. E., Barral, A. M., Rodrigo, E., Oldstone, M. B., von Herrath, M. G. and Christen, U. (2005). Islet-specific expression of CXCL10 causes spontaneous islet infiltration and accelerates diabetes development. J Immunol, 175, 3516-3524.

Rollins, B. J. (1991). JE/MCP-1: an early-response gene encodes a monocyte-specific cytokine. Cancer Cells, 3, 517-524.

Rollins, B. J., Walz, A. and Baggiolini, M. (1991). Recombinant human MCP-1/JE induces chemotaxis, calcium flux, and the respiratory burst in human monocytes. Blood, 78, 1112-1116.

Rot, A. and von Andrian, U. H. (2004). Chemokines in innate and adaptive host defense: basic chemokinese grammar for immune cells. Annu Rev Immunol, 22, 891-928.

Siveke, J. T. and Hamann, A. (1998). Thelper 1 and T helper 2 cells respond differentially to chemokines. J Immunol, 160, 550-554.

Soejima, K. and Rollins, B. J. (2001). A functional IFN-gamma-inducible protein-10/CXCL10specific receptor expressed by epithelial and endothelial cells that is neither CXCR3 nor glycosaminoglycan. J Immunol, 167, 6576-6582.

Stiles, L. N., Hardison, J. L., Schaumburg, C. S., Whitman, L. M. and Lane, T. E. (2006). T cell antiviral effector function is not dependent on CXCL10 following murine coronavirus infection. J Immunol, 177, 8372-8380.

Tani, M., Fuentes, M. E., Peterson, J. W., Trapp, B. D., Durham, S. K., Loy, J. K., Bravo, R., Ransohoff, R. M. and Lira, S. A. (1996). Neutrophil infiltration, glial reaction, and neurological disease in transgenic mice expressing the chemokine N51/KC in oligodendrocytes. J Clin Invest, 98, 529-539.

Taub, D. D., Lloyd, A. R., Wang, J. M., Oppenheim, J. J. and Kelvin, D. J. (1993). The effects of human recombinant MIP-1 alpha, MIP-1 beta, and RANTES on the chemotaxis and adhesion of T cell subsets. Adv Exp Med Biol, 351, 139-146.

Taub, D. D., Longo, D. L. and Murphy, W. J. (1996a). Human interferon-inducible protein-10 induces mononuclear cell infiltration in mice and promotes the migration of human $\mathrm{T}$ lymphocytes into the peripheral tissues and human peripheral blood lymphocytes-SCID mice. Blood, 87, 1423-1431.

Taub, D. D., Ortaldo, J. R., Turcovski-Corrales, S. M., Key, M. L., Longo, D. L. and Murphy, W. J. (1996b). Beta chemokines costimulate lymphocyte cytolysis, proliferation, and lymphokine production. J Leukoc Biol, 59, 81-89.

Toft-Hansen, H., Buist, R., Sun, X. J., Schellenberg, A., Peeling, J. and Owens, T. (2006). Metalloproteinases control brain inflammation induced by pertussis toxin in mice overexpressing the chemokine CCL2 in the central nervous system. J Immunol, 177, 7242-7249. 
Ubogu, E. E., Cossoy, M. B. and Ransohoff, R. M. (2006). The expression and function of chemokines involved in CNS inflammation. Trends Pharmacol Sci, 27, 48-55.

Vlkolinsky, R., Siggins, G. R., Campbell, I. L. and Krucker, T. (2004). Acute exposure to CXC chemokine ligand 10, but not its chronic astroglial production, alters synaptic plasticity in mouse hippocampal slices. J Neuroimmunol, 150, 37-47.

Yamamoto, M., Horiba, M., Buescher, J. L., Huang, D., Gendelman, H. E., Ransohoff, R. M. and Ikezu, T. (2005). Overexpression of monocyte chemotactic protein-1/CCL2 in beta-amyloid precursor protein transgenic mice show accelerated diffuse beta-amyloid deposition. Am $J$ Pathol, 166, 1475-1485. 